in 13 and symptomatic in 31 infants. Vigabatrin was the initial treatment in 36 , and all infants were followed until 12 months of age. On the first video-EEG before treatment, 27 infants showed hypsarrhythmia, and 17 had multifocal spikes with normal EEG background. A 4-hour recording including waking, sleep, and awakening was usually sufficient to detect spasms. Subtle, asymmetric or asynchronous spasms, and asymmetric ictal or interictal EEG abnormalities were associated with symptomatic etiology and poor cognitive and seizure outcome. Treatment modifies both spasms and EEG; spasms may become subtle and only discernible on EEG, and hypsarrhythmia may be replaced by multifocal spikes. Hypasarrhythmia rarely persists following vigabatrin treatment. Complete eradication of spasms, including subtle variants, is essential for a favorable cognitive outcome. (Gaily E, Liukkonen E, Paetau R et al. Infantile spasms: diagnosis and assessment of treatment response by video-EEG. Dev Med Child Neurol Oct 2001;43:658-667). (Respond: Dr Eija Gaily, PO Box 280, Hospital for Children and Adolescents, FIN-00029 HYKS, Finland).

COMMENT. Video-EEG may be essential for the initial diagnosis of infantile spasms, especially in infants with symptomatic etiology and subtle spasms. It is also necessary in assessing response to treatment and the exclusion of persisting subtle spasms not clinically evident.

\title{
RISK FACTORS FOR EARLY NEONATAL SEIZURES
}

Prenatal and perinatal risk factors for neonatal seizures occurring in 100 newborns in the first week were determined in a case-control study at Grottaferrata, the University of Rome, and other centers, Italy. The majority $(71 \%)$ had more than one type of seizure, the most common being generalized tonic (29\% of infants). Less frequently, multifocal clonic (13\%), focal clonic (9\%), and myoclonic seizures (5\%); all were associated with subtle seizures. Interictal EEG was normal in $20 \%$, and showed asymmetries of background activity in $47 \%$, burst suppression in 5\%, and episodic voltage attenuation in $28 \%$. Etiologies presumed to be responsible for seizures included hypoxic-ischemic encephalopathy in 30\%, intracranial hemorrhage $(10 \%)$, hypocalcemia $(9 \%)$, meningitis $(8 \%)$, hypoglycemia $(2 \%)$, and unknown $(10 \%)$. A history of epilepsy in first degree relatives was found in 4 cases and for none of the controls. Parents of cases had a lower socioeconomic status than those of controls. Neonatal seizures were associated with maternal disease in the 2 years preceding the pregnancy, excessive weight gain, placental pathology, preeclampsia, low geastational age, low birth weight, and neonatal jaundice. Cardiopulmonary resuscitation was needed in $37 \%$ of cases and none of the controls. (Arpino C, Domizio S, Carrieri MP et al. Prenatal and perinatal determinants of neonatal seizures occurring in the first week of life. I Child Neurol October 2001;16:651-656). (Respond: Dr Carla Arpino, E Litta Rehabilitation Center for Developmental Disabilities, Via Anagnina Nuova, 13, 00046 Grottaferrata, Rome, Italy).

COMMENT. Neonatal seizures in the first week are strongly associated with low gestational age and low birthweight, risk factors that might be preventable. Other familial. maternal, and neonatal factors are associated, including placental pathology, preeclampsia, and a family history of epilepsy and febrile seizures.

\section{KETOGENIC DIET IN REFRACTORY FOCAL SEIZURES}

The efficacy of the ketogenic diet in the treatment of intractable focal seizures was studied retrospectively in 34 patients (mean age, $7.5 \mathrm{yrs}$; range, 4 months to $29 \mathrm{yrs}$ ) and compared to 100 patients with generalized seizures at the 